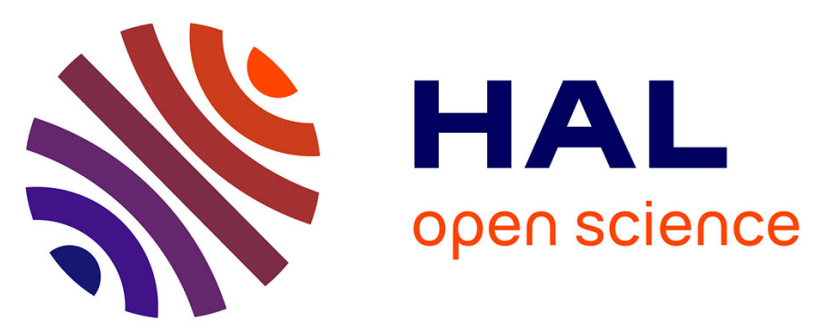

\title{
Optical system with a curved detector for wide-field high-resolution cortical imaging at meso-scale
}

Isabelle Racicot, Eduard Muslimov, Xavier Degiovanni, Joel Baurberg, Kévin Blaize, Jean-François Sauvage, Marc Ferrari, Frédéric Chavane

\section{- To cite this version:}

Isabelle Racicot, Eduard Muslimov, Xavier Degiovanni, Joel Baurberg, Kévin Blaize, et al.. Optical system with a curved detector for wide-field high-resolution cortical imaging at meso-scale. SPIE Optical Systems Design 2021, Sep 2021, Online, Spain. pp.118760A, 10.1117/12.2597067 . hal03441839

\section{HAL Id: hal-03441839 \\ https://hal-amu.archives-ouvertes.fr/hal-03441839}

Submitted on 22 Nov 2021

HAL is a multi-disciplinary open access archive for the deposit and dissemination of scientific research documents, whether they are published or not. The documents may come from teaching and research institutions in France or abroad, or from public or private research centers.
L'archive ouverte pluridisciplinaire HAL, est destinée au dépôt et à la diffusion de documents scientifiques de niveau recherche, publiés ou non, émanant des établissements d'enseignement et de recherche français ou étrangers, des laboratoires publics ou privés. 


\title{
Optical system with a curved detector for wide-field high-resolution cortical imaging at meso-scale.
}

\author{
Isabelle Racicot ${ }^{\mathrm{a}, \mathrm{b}}$, Eduard Muslimov ${ }^{\mathrm{d}, \mathrm{e}, \mathrm{b}}$, Xavier Degiovanni ${ }^{\mathrm{a}}$, Joel Baurberg ${ }^{\mathrm{a}}$, Kévin Blaize ${ }^{\mathrm{a}}$, \\ Jean-François Sauvage ${ }^{c, b}$, Marc Ferrari $^{\mathrm{b}}$, and Frédéric Chavane ${ }^{\mathrm{a}}$ \\ anstitut de Neurosciences de la Timone : Aix-Marseille Univ, CNRS, INT, Marseille, France \\ ${ }^{\text {b}}$ Laboratoire d'Astrophysique de Marseille : Aix-Marseille Univ, CNRS, CNES, LAM, \\ Marseille, France \\ 'ONERA, Département d'Optique Théorique et Appliquée (DOTA), Chatillon, France \\ ${ }^{d}$ NOVA Optical IR Instrumentation Group at ASTRON Oude Hoogeveensedijk 4, 7991 PD \\ Dwingeloo, The Netherlands

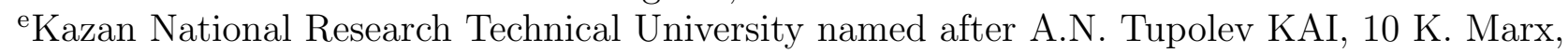 \\ Kazan, Russia, 420111
}

\begin{abstract}
Optical imaging techniques such as voltage-sensitive dye imaging and intrinsic imaging allow for the recording of neuronal activity at high spatio-temporal scales over a large field of view ${ }^{1},{ }^{2}$ revealing some mesoscopic scale dynamics such as propagating waves ${ }^{3}{ }^{4}$ In practice however, the achievable image quality deteriorates significantly away from the point of best focus due to the curvature of the brain, which fundamentally limits the spatial extent of the cortex that can be studied through a single image. To improve the field of view achievable by optical imaging, we developed a new optical system adapted to the curvature of the non-human primate brain in study. This is achieved by using a curved detector in combination with an appropriate optical system of double Gaussian and aspherical lenses. Furthermore, to ensure a uniform and reliable illumination of the cortex, we have designed and built a new illumination system consisting of a ring of LEDs at four different wavebands. This static solution will enable imaging for the first time neuronal activity over a very large field of view (15-20mm) with high spatial and temporal resolution. Preliminary results show a significant increase of the area in focus of a spherical object imaged through the custom optics compared with the standard neuronal imaging optics.
\end{abstract}

Keywords: Curved detector, neuroscience, cortical imaging, large field of view

\section{INTRODUCTION}

Numerous studies attempt to comprehend the finer inner processing of the brain, which is not fully understood. Several techniques allow to measure cortical activity at different scales and various levels of precision, from the macroscopic scale magnetic resonance imaging (MRI) to microscopic scale intracellular recordings. The ideal neuronal recording technique would combine access to a large field of view with a high spatio-temporal resolution. ${ }^{2}$ In practice there is generally a trade-off between the field of view and the resolution, which limits the kind of cortical activity that can be observed. One such behavior of interest are cortical travelling waves, which are thought to impact on the way the brain processes information. The observation of these propagating waves requires recording cortical responses over several cortical areas at high spatial and temporal resolutions. Such waves have been observed with electrode arrays, in macaques' visual cortex ${ }^{5}$ in primary motor cortex (M1) and premotor cortex $(\mathrm{PMd})^{6}$ and in marmoset middle temporal visual (MT) area. ${ }^{7}$ However this technique is inherently limited by the spatial extent of the cortex that can be measured, as it is not quite large enough to capture all of the cortical travelling waves, and by the fact that the measurement is done with discrete recording sites (the electrodes) such that the extracted signal is discontinuous and subject to sampling problems. Optical imaging techniques like intrinsic imaging (based on the fact that oxygenated and deoxygenated hemoglobin in

Further author information: (Send correspondence to I. Racicot)

e-mail: isabelle.racicot@univ-amu.fr 
the blood have different absorption levels) and voltage sensitive dye imaging (VSDI - using a dye that emits a fluorescence depending on the neighbouring neurons' voltage) offer the possibility to continuously image a large field of view (up to $2 \mathrm{~cm}$ ) with high spatial and temporal resolution (on the order of $50 \mu \mathrm{m}$ and 1-10 ms, respectively ${ }^{2}$. Indeed, cortical travelling waves have been observed through VSD imaging, from the anesthetized turtle cortex ${ }^{8}$ to the awake macaque. ${ }^{9}$ In both these cases, clever analysis of noisy data have been performed in order to reveal the wave pattern. This is because VSDI signal is often quite weak and noisy, and is usually averaged over several trials in order to strengthen the signal to noise ratio. Since this averaging also washes out finer details of the response, such as wave patterns, it becomes preferable to avoid averaging and work with single trials.

For these reasons, improving the image field of view and signal to noise ratio that can be achieved by optical imaging techniques would greatly facilitate the observation of some neuronal responses like travelling waves. The element that at the present mostly restricts the imaging capacities is the curvature of the brain that is observed. Because of it, the area in focus in any given image is limited to the central area whilst the image quality decreases sharply away from the centre as it moves out of focus. The result is that although a large cortical area is technically available for imaging, only a subsection of it can be properly imaged (approximately half of the imaged region). Attempts to increase the depth of focus by reducing the aperture of the imaging system is counterproductive since it lowers the amount of light that reaches the detector, thus making a low signal even fainter. Similarly, increasing the light intensity too much is also undesirable because of possible photodynamic damage and too rapid bleaching from the florescence dye. Our solution to this issue is to design and build an optical imaging system adapted to the curvature of the studied brain such that most points in the available $15-20 \mathrm{~mm}$ field of view are imaged in focus.

\section{SETUP}

The starting point of this project was the optical setup in use at the Institut de Neurosciences de la Timone (INT), manifactured by Optical Imaging Ltd. It is composed of two $50 \mathrm{~mm}$ Pentax objectives mounted front to front. The camera (Photonfocus MV1-D1312) includes a flat CMOS detector that has $1312 \times 1082$ pixels with dimensions of $8.0 \mu \mathrm{m} \times 8.0 \mu \mathrm{m}$ (active area of $10.48 \mathrm{~mm} \times 8.64 \mathrm{~mm}$ ), with a greyscale resolution of 12 bits and a frame rate of $108 f$ fps. The illumination is provided by a $100 \mathrm{~W}$ halogen lamp (HAL-100) and its light is filtered at the selected wavelength by an optical filter. For intrinsic imaging, the light is fibre coupled into two large multi-mode optical fibres. The output of each fibre is held by an articulated arm and manually positioned before each procedure to optimize the uniformity of the illumination on the cortex. For VSD imaging, the fluorescent signal is emitted at a different wavelength and can thus be filtered our from the illuminating light. Making use of this fact, a dichroic mirror is added between the two objectives and the light emitted by the lamp is reflected from this mirror towards the brain (epi-illumination through the objective), while the light emitted by the dye goes through the dichroic mirror to reach the camera. This imaging instrument can be used in various settings (with mice, marmosets, macaques, etc.), but this project focuses on the imaging of the primary visual cortex of the rhesus macaque. The brain is imaged through a custom-made $20 \mathrm{~mm}$ wide optical chamber. The natural dura mater is replaced by an artificial dura mater which can me temporarily removed for VSD imaging preparation (staining of the dye). The chamber is fixed to the animal's skull and filled with a transparent agar solution. The chamber is then closed by a perspex window and the imaging is done through the closed chamber filled with agar.

The development of the new optical setup has been realized on a optical test bench located at the Laboratoire d'Astrophysique de Marseille (LAM). In addition to the new optical setup, an imaging system identical to the one in use at INT (with the exception of the halogen lamp) has also been built on the same optical bench. A photograph of this reproduced instrument is shown in figure 1. This allows testing of both imaging tools in identical conditions and facilitates the characterization of the initial flat sensor setup, which would otherwise be challenging due to overlapping neuroscience experiments and a somewhat limited physical access.

In order to develop the new setup, several test objects have been 3D printed. For most testing purposes, the imaged objects were spheres that were $3 \mathrm{D}$ printed at the proper curvature, as determined from the macaque brain's curvature (see section 2.1). Specifically, two cases were considered: the "dry" setup, in which the curved test object is imaged directly as is, as opposed to the "wet" setup, in which the test curved object is 


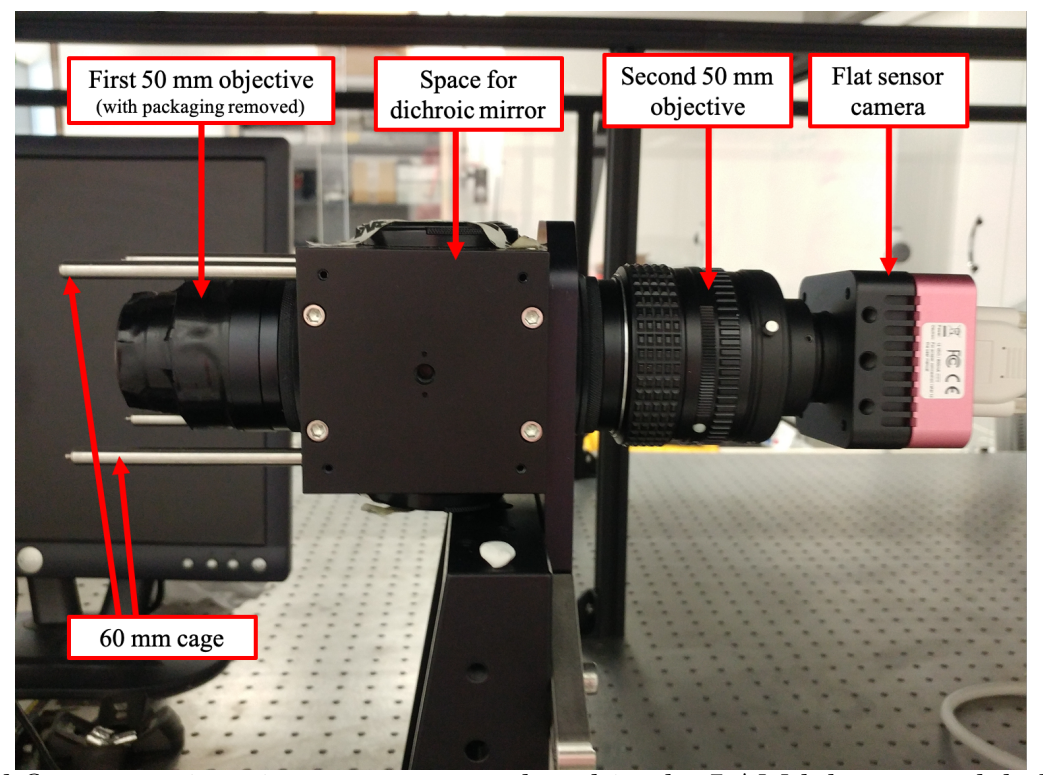

Figure 1: The original flat sensor imaging system reproduced in the LAM laboratory, labeled. It consists of two objectives (one with its packaging removed to reduce its size) and a flat sensor camera.

imaged through the agar-filled optical chamber. The target radius of curvature in both cases is different: this is because agar, which has an index of refraction similar to that of water, is spread out between the flat optical chamber's window and the curved object, and therefore acts as an extra lens. While the "wet" setup is closer to the experimental conditions at INT, the presence of the chamber and agar adds extra complexity and so for the initial testing stages, the "dry" setup was used. The characterization of the imaging system presented in section 3 was done in these conditions.

Several options were considered to create a pattern on the 3D printed object in order to observe a meaningful image. In the end, simplicity prevailed and the sphere was painted white and small, 0.25 mm diameter dots were added to the surface in black ink with a precision pen. Although imperfect, the resulting pattern offers sharp edges, high contrast and can be easily and quickly added to most surfaces.

\subsection{Detector and Camera}

The first element of the new optical setup is the detector, since its size and how much it can be curved determine the rest of the optical system. The detector that was chosen for this project is a greyscale, 12 bit CMOS detector (AMS CMV12000). It has 4096x3072 square pixels with a width of $5.5 \mu \mathrm{m}$, an active area of $22.5 \mathrm{~mm} \times 16.9 \mathrm{~mm}$ and a frame rate of up to $132 \mathrm{fps}$ at full resolution and full bit depth. This kind of detector was selected because it can by curved by a technique based on substrate elasticity to a specific radius of curvature be Curve-One, a startup stemming from the Laboratoire d'Astrophysique de Marseille (LAM).

Before any curving of the detector can be done, the target radius of curvature must be determined. This radius of curvature represents the global curvature of the cortical surface in the area that is studied through optical imaging. The curvature is estimated by analyzing data from magnetic resonance imaging (MRI) scans that were carried out on several rhesus macaque monkeys. The cortical surface is extracted from this data through an open-source, Python-based MRI segmentation pipeline, Macapype. The resulting surface is further analysed through Anatomist, a visualizing software that is part of BrainVISA, an open-source neuroimaging software platform. In this step, a region of interest is defined, corresponding to the cortical area that is imaged through the optical chamber (in this case, the primary visual cortex). The points forming the area of interest are extracted and a spherical surface is fit to these points to obtain the area's estimated radius of curvature. This analysis has been performed on several monkeys in order to get a sample of the variations that can be expected between individuals. ${ }^{10}$ Ultimately, the target curvature that was selected $(28.89 \mathrm{~mm})$ corresponds to the brain 
curvature of the macaque currently working at INT but fits well within the distribution of brain curvatures as determined by the fits (between $20 \mathrm{~mm}$ and $35 \mathrm{~mm}$ ).

The curving technology does not allow to curve the detector to such a small radius of curvature. As a consequence, the detector was curved to the smallest radius that could be safely attained $(160 \mathrm{~mm})$ and the rest of the curvature is accounted for by the optics (see section 2.2).

The detector must be used in combination with a camera in order to obtain any image. Note that we refer to the detector and the camera as distinct components: in this case, the detector refers to the photosensitive surface (the sensor itself) and the camera refers to the rest of the electronic components. The distinction arises from the fact that in this system these two elements, detector and camera, are completely independent and can be freely swapped around. The camera that is used with the CMOS detector in this project is the apertus ${ }^{\circ} \mathrm{AXIOM}$ Beta Developer Kit, which is an open source camera that lets users choose their own detectors. The camera freed from its (home-made) enclosure, along with our two curved detectors, is show in figure 2. Once the camera is connected to the internet through an Ethernet cable it can receive commands, take and send images to a computer through a secure shell protocol (ssh) connection.

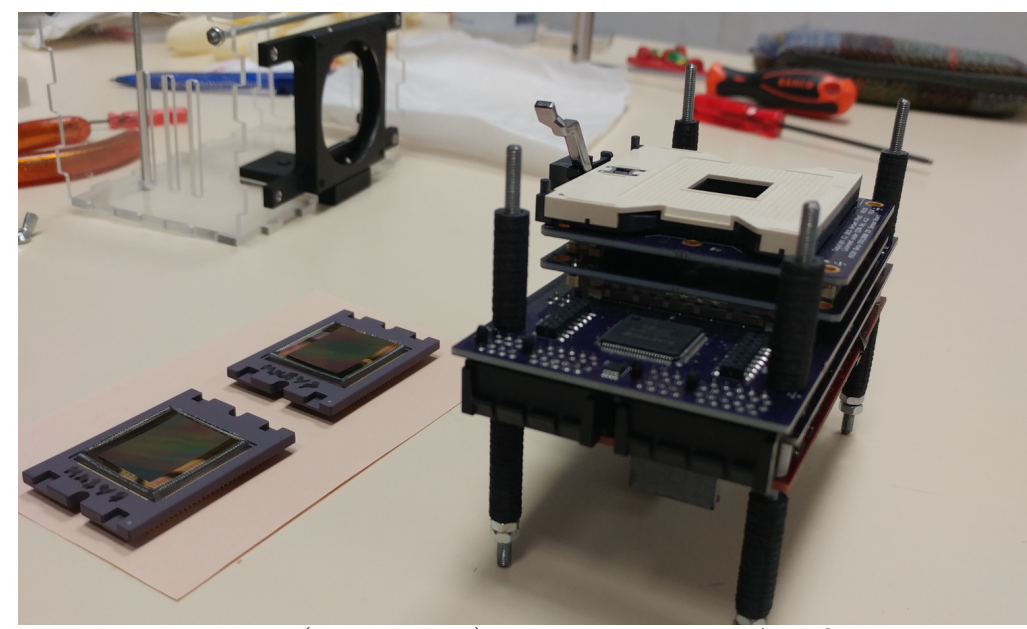

Figure 2: The two curved detectors $(\mathrm{R}=160 \mathrm{~mm})$ and the apertus ${ }^{\circ} \mathrm{AXIOM}$ Beta Developer Kit camera

\subsection{Optics and Lens Mount}

The second part of the optical system is the optics to be used in combination with the detector. As explained in section 2.1, a large amount of the brain curvature must be accounted for by the lenses themselves because of the curving process' limitations. The design of the optics was done by optimization in Zemax's Optics Studio software. ${ }^{10}$

The resulting optical design consists of a traditional double Gauss unit combined with an aspherical lens that corrects for the rest of the curvature not accounted for by the detector. The resulting 7 lenses have non-standard physical dimensions, such that mounting the optical system using off the shelf mounts was unpractical. It was therefore decided to design a custom mount for the optics. Several designs with various degrees of freedom were considered; concerns of time, technical availability and required precision lead us to select a mount design consisting of three main components, that we subcontracted to a company specialised in precision 3D printing. The design simplifies the assembly while still offering several degrees of freedom to optimize the alignment of sensitive elements. The lenses are maintained in place by locking rings and the spacing between them is determined by separating cones designed to fit exactly inside the mount's elements. The lens enclosure can be mounted on a Thorlabs cage system, on which the illumination system is also placed (see section 4), and it screws directly into the E-mount of the camera. The 3D printed pieces of the mount can be seen in figure 3, and the assembled setup, with the mount, the lenses, the camera and the illumination system is shown in figure 4. A sketch of the mount's cross section is shown in figure 5 . 


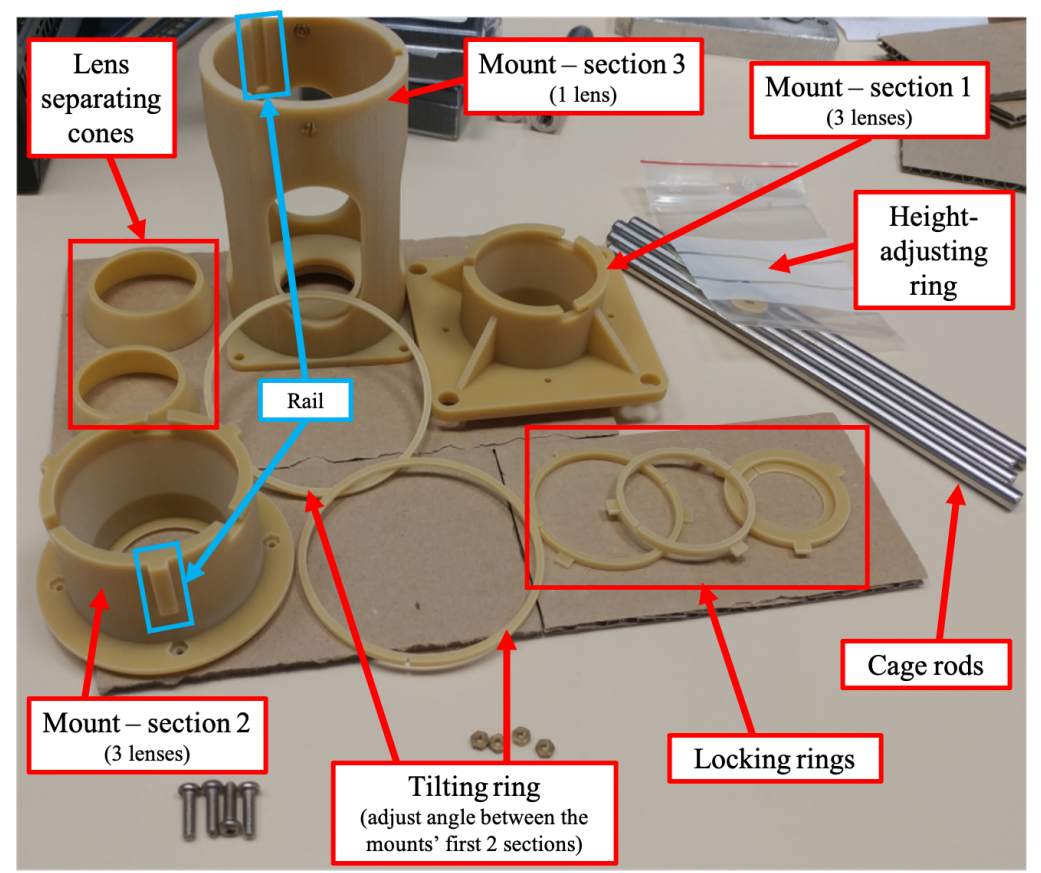

Figure 3: The eleven 3D printed pieces of the lens mount, labeled. The two first sections of the mount contain the Double Gauss lenses, separated by special cones, and the angle between the two sections can be tweaked by stacking two "tilting rings" of slightly uneven thickness. The third section contains the aspherical lens and screws directly on the camera's E-mount. A small rail between sections 2 and 3 (labeled in blue) allows to adjust the distance between the aspherical lens and the Double Gauss. The finished mount can me installed on a 60 mm cage system.

\section{OPTICAL PERFORMANCE CHARACTERIZATION}

Once the new, curved detector setup has been put together, the question of quantifying its optical performances arises. The sections below describe measurements that were done in order to characterize the resulting optical system and to compare it with both the expected performance and the original, flat sensor setup.

\subsection{Point Spread Function Measurement}

The setup is characterized by measuring the point spread function (PSF) at different positions in the imaging field. The PSF is measured by imaging a point-like source created by a $2 \mu \mathrm{m}$ pinhole illuminated with red light $(670 \mathrm{~nm})$. The red light is produced by a laser, so to avoid interference effects the light is first sent through a diffusing plate before reaching the pinhole. Since the setup is optimized for a curved object, the PSF must be measured at different points along the surface of a sphere. This is achieved by placing the pinhole on a translation stage which can be moved precisely along the 3 axis. We move the pinhole by steps of $2 \mathrm{~mm}$ (vertical axis, "y") and $4 \mathrm{~mm}$ (horizontal axis "x") and calculate for each of these points the exact position along the optical axis ("z") such that the pinhole scan follows the surface of a sphere with the appropriate curvature. Resulting PSFs are shown in figure 6 for both the curved and flat detector setups. The positions in the detector plane of these PSFs are shown in figure 7. Note that the flat sensor camera is angled by $6.5^{\circ}$ with respect to the object plane, such that the scanned area appears tilted in the figures. The angle is caused by the fact that the camera is screwed into a static mount, which does not allows adjustments. The original flat sensor imaging system has a magnification of 1 and the curved sensor system has a theoretical magnification of 0.893 , which has been confirmed experimentally.

In order to quantify the performance of each optical system and compare them to each other, the measured PSFs were fitted to a two dimensional Gaussian function and values for the full width half max (FWHM) size along the short and long axis of the spot were extracted from those fits. The results are show in figure 8a. The 


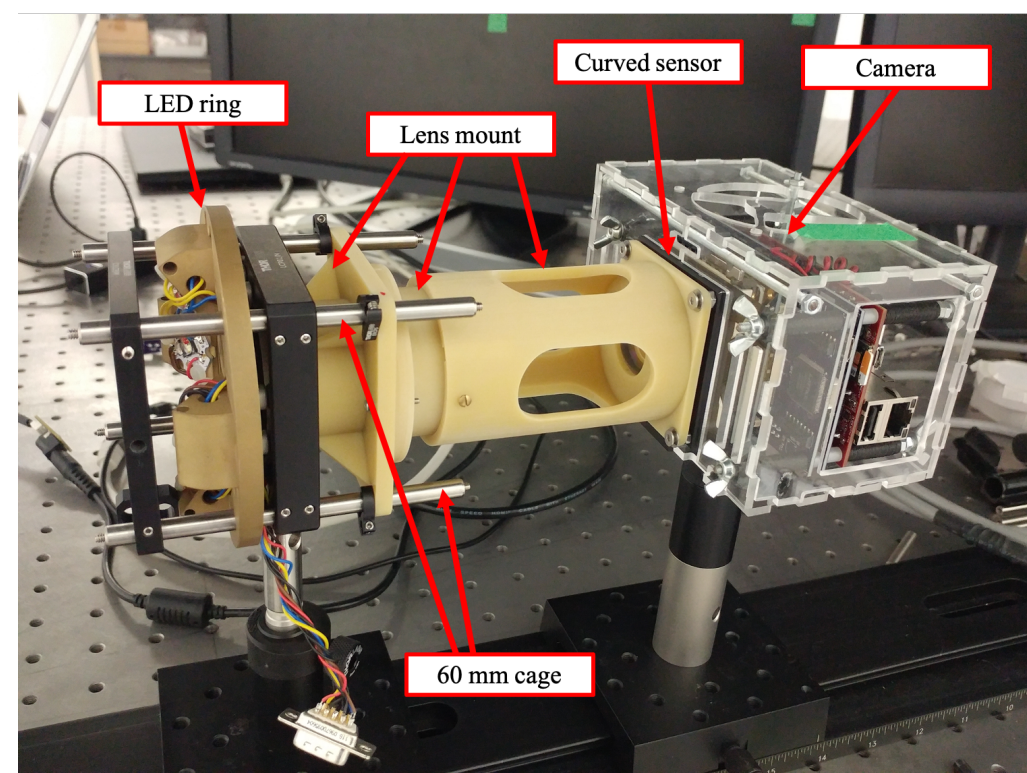

Figure 4: The curved sensor optical imaging instrument, labeled. From left to right: the LED ring, the 3D printed mount containing the seven lenses, and the apertus ${ }^{\circ}$ AXIOM Beta camera with its custom enclosure.

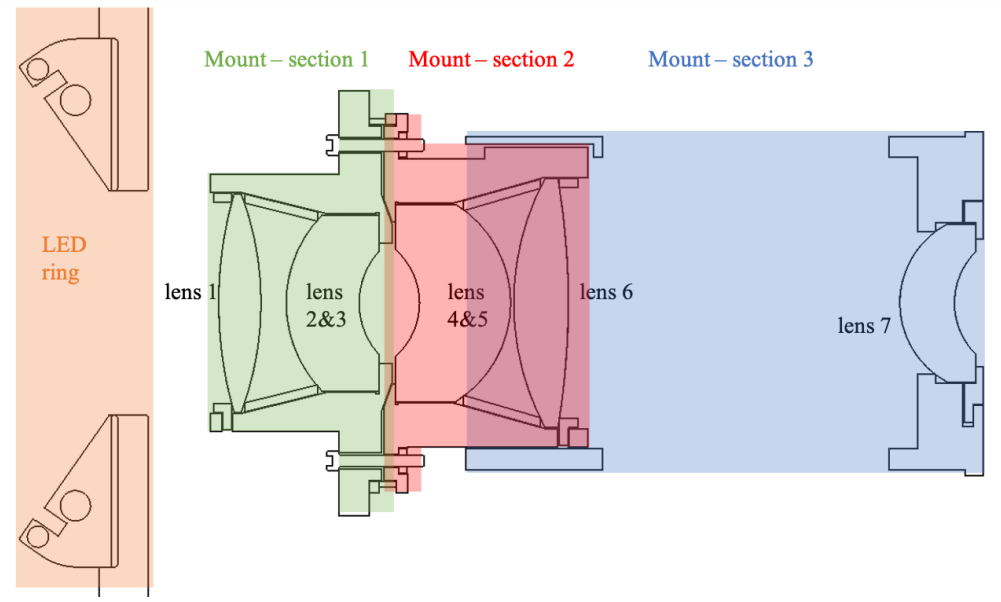

Figure 5: Sketch of the mount's cross-section with the LED ring. The lenses are labelled and the three sections of the mount are illustrated by colored blocks (green, red and blue). The outline of the locking rings and the separating cones can be seen near the edges of the lenses. Lenses 1 to 6 are the double Gauss lenses (because they are so close to each other, lenses 2 and 3 as well as lenses 4 and 5 were glued together during manufacturing) and lens 7 is the aspherical lens.

improvement achieved by changing from the original, flat detector setup to the new, curved detector setup is shown for different positions away from the centre in figure $8 \mathrm{~b}$ and range from negligible $(<1 \%)$ at the centre to over $80 \%$ for the furthest points that could be compared. Since the curved detector setup has a much larger field of view, only the points closer to the centre could be compared to those obtained with the flat detector.

A theoretical spot size for one quadrant of the positions measured was estimated through non sequential simulations with the simulation software that was used for the optical design (Zemax's Optics Studio). The main results for the bottom left quadrant are summarized in table 1. The comparison between the experimental and expected results holds relatively well at the centre but the measured spot size becomes significantly larger for the most distant points (for radial positions greater than $8 \mathrm{~mm}$ ). While it could be caused by possible misalignment of the optics, the shape of the PSF at the points leads us to suspect that the presence of parasite light reflected from the various surfaces of the lens mount could be creating the observed pattern. The fact the 


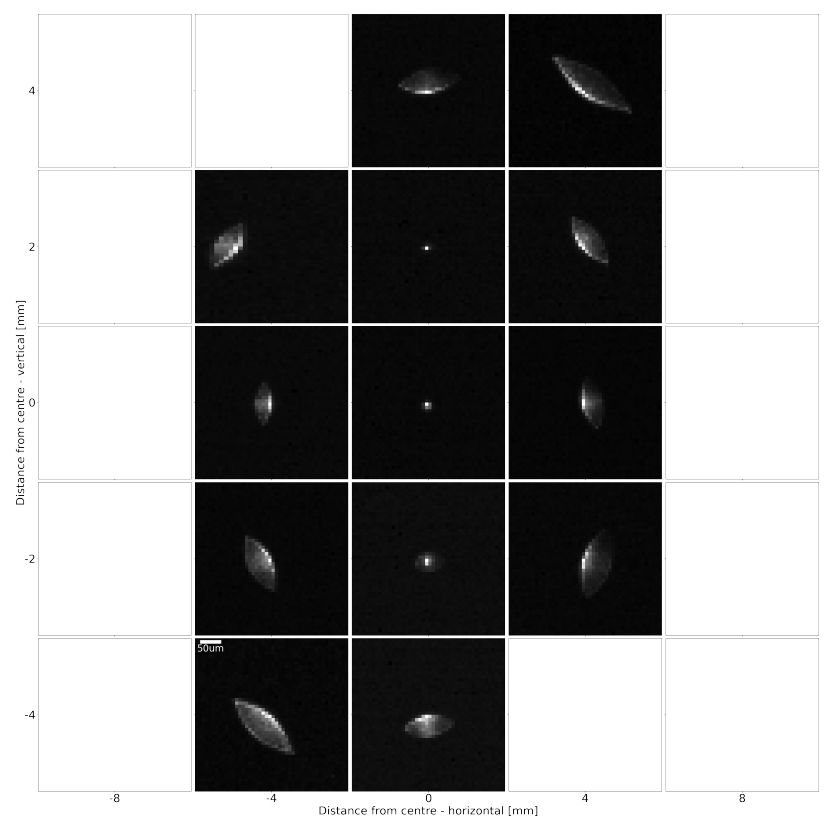

(a) Original (flat) detector setup
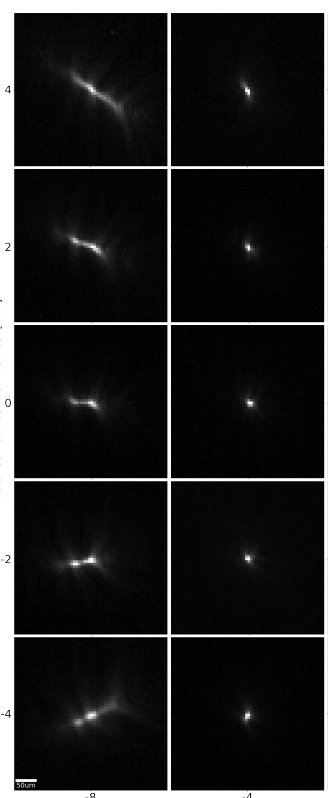

(b) Curved detector setup

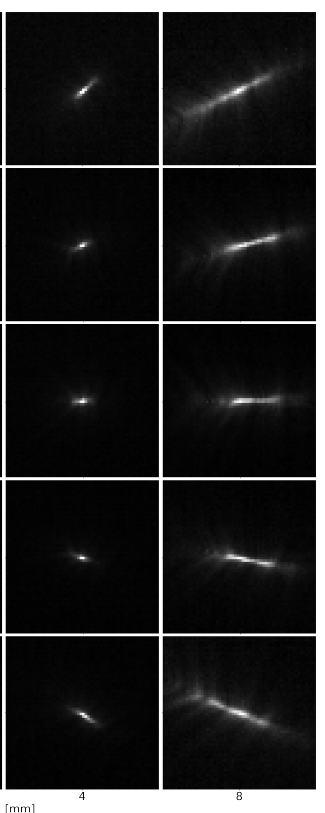

Figure 6: PSF measured on the detector area by scanning a $2 \mu \mathrm{m}$ pinhole illuminated by red light across the field of view. Points along the horizontal axis are separated by $4 \mathrm{~mm}$, those along the vertical axis are separated by 2 $\mathrm{mm}$. The PSF subimages have a size of $0.37 \mathrm{~mm}$ for both the curved and flat detector setups (60 curved pixels, 46 flat pixels). Since the flat detector is smaller than the curved detector, not all points could be measured for this setup, resulting in several empty subimages. Note that the flat sensor camera is angled by $6.5^{\circ}$ with respect to the object coordinate system.

these points lie just outside of the field for which the system was optimized also makes such simulations trickier.

\begin{tabular}{|c|c|c|c|c|c|c|c|c|c|}
\hline \multirow{2}{*}{\multicolumn{2}{|c|}{ Position }} & \multicolumn{4}{|c|}{ Curved detector } & \multirow{2}{*}{\multicolumn{2}{|c|}{$\begin{array}{c}\text { Flat detector } \\
\text { Measured FWHM }\end{array}$}} & \multirow{2}{*}{\multicolumn{2}{|c|}{$\begin{array}{c}\text { Improvement } \\
\text { On measured FWHM }\end{array}$}} \\
\hline & & Theoretic & spot size & Measure & FWHM & & & & \\
\hline $\begin{array}{c}\mathrm{X} \\
{[\mathrm{mm}]}\end{array}$ & $\begin{array}{c}\mathrm{Y} \\
{[\mathrm{mm}]}\end{array}$ & $\begin{array}{l}\text { short axis } \\
{[\mu \mathrm{m}]}\end{array}$ & $\begin{array}{c}\text { long axis } \\
{[\mu \mathrm{m}]}\end{array}$ & $\begin{array}{l}\text { short axis } \\
{[\mu \mathrm{m}]}\end{array}$ & $\begin{array}{c}\text { long axis } \\
{[\mu \mathrm{m}]}\end{array}$ & $\begin{array}{c}\text { short axis } \\
{[\mu \mathrm{m}]}\end{array}$ & $\begin{array}{c}\text { long axis } \\
{[\mu \mathrm{m}]}\end{array}$ & $\begin{array}{c}\text { short axis } \\
{[\%]}\end{array}$ & $\begin{array}{c}\text { long axis } \\
{[\%]}\end{array}$ \\
\hline 0.20 & 0.17 & 23.23 & 23.23 & 17.53 & 18.72 & 18.84 & 18.84 & 6.95 & 0.64 \\
\hline 0.17 & 2.21 & 17.39 & 19.27 & 13.44 & 19.57 & 18.84 & 20.74 & 28.66 & 5.64 \\
\hline 0.16 & 4.24 & 6.48 & 10.99 & 15.64 & 28.57 & 39.76 & 49.32 & 60.66 & 5.64 \\
\hline-3.89 & 0.14 & 8.13 & 12.36 & 13.33 & 18.04 & 29.64 & 41.84 & 55.03 & 56.88 \\
\hline-3.93 & 2.19 & 8.02 & 13.96 & 13.92 & 17.89 & 38.79 & 70.59 & 64.11 & 74.66 \\
\hline-3.96 & 4.24 & 8.52 & 15.60 & 12.88 & 20.94 & 46.65 & 112.17 & 69.26 & 82.43 \\
\hline-8.26 & 0.11 & 9.55 & 11.86 & 18.80 & 74.06 & - & - & - & - \\
\hline-8.34 & 2.24 & 8.78 & 15.58 & 18.40 & 79.23 & - & - & - & - \\
\hline-8.47 & 4.39 & 8.23 & 12.54 & 26.68 & 101.54 & - & - & - & - \\
\hline
\end{tabular}

Table 1: Comparison between experimental measurements (white for curved detector setup and light blue for the original flat detector setup) and theoretical expectations for the curved detector setup (light gray) of the PSF spot size at different positions in the image field. The improvement in percentage at each field position between the original setup and the new curved detector setup is shown in the last two columns (light yellow). The theoretical spot sizes were computed by simulation of the whole setup in Zemax's Optics Studio software. The spot size is given along the short and long axis for all points. No measurements could be taken at larger field eccentricities with the flat detector setup due to the fact that the flat detector has a smaller surface (10.48 $\mathrm{mm} \times 8.64 \mathrm{~mm})$ than the curved detector $(22.5 \mathrm{~mm} \times 16.9 \mathrm{~mm})$. 


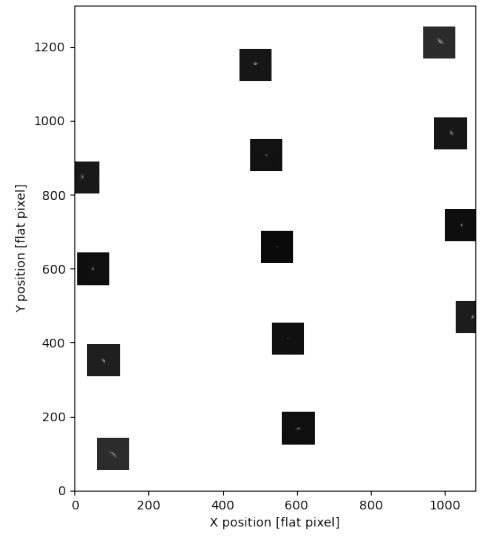

(a) Original (flat) setup

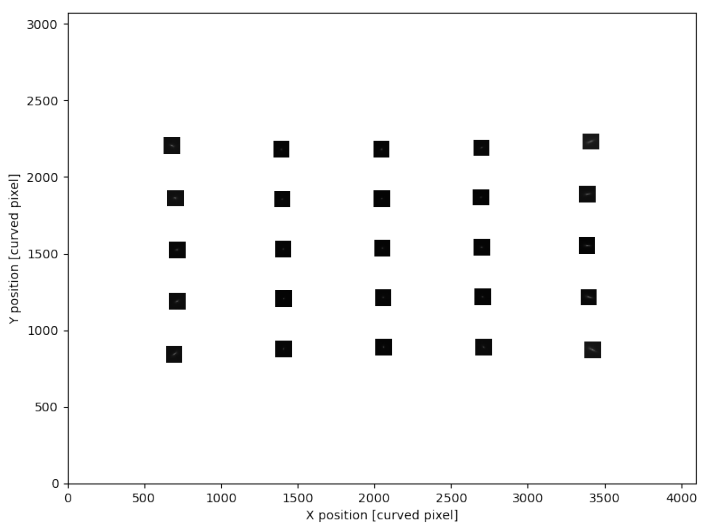

(b) Curved detector setup

Figure 7: Positions of the measured PSFs on the sensor area for each setup. Points along the horizontal axis are separated by $4 \mathrm{~mm}$ and those along the vertical axis are separated by $2 \mathrm{~mm}$. The subimages (dark squares) have a width and height of $0.37 \mathrm{~mm}$ for both the curved and flat detector setups. Note that the camera with the flat sensor has an angle of $6.5^{\circ}$ with respect to the object coordinate system.

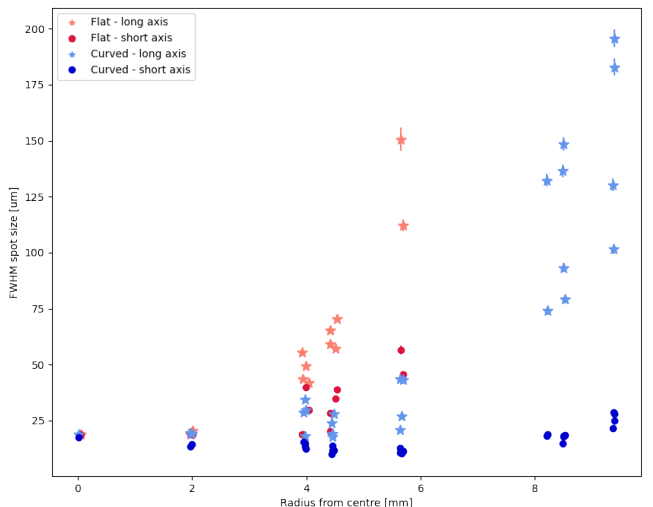

(a) Fitted spot size (FWHM)

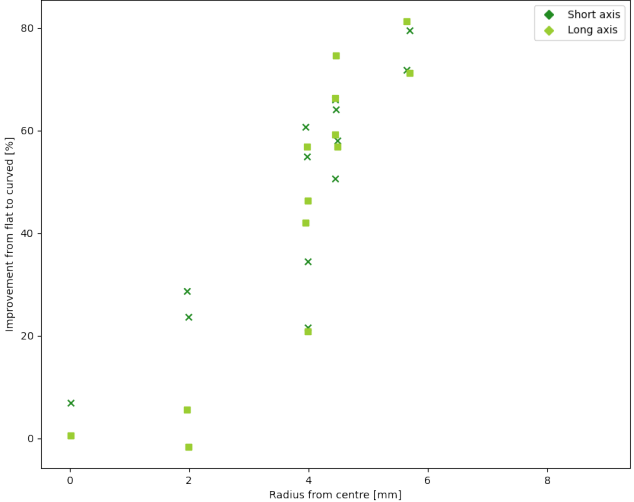

(b) Improvement between the two imaging systems

Figure 8: Spot size measured experimentally for both the original, flat detector setup and the new, curved detector setup. The spot size, determined by fitting the PSF data to a 2D Gaussian, is given in $\mu \mathrm{m}$ along both the long and short axis. The radial distance from the centre of the detector (in $\mathrm{mm}$ ) is determined from the $\mathrm{x}$ and y positions of the PSF's brightest point. In figure a, error bars show the estimated standard deviation error on the fit. Figure b shows for each radial position the improvement from the original to the new setup, for points where values exist for both setups, given as a percentage of the original spot size.

The spot size in the area between and beyond the sampled points was estimated by fitting a polynomial function assuming radial symmetry to the data and applying this fit function to the area of interest. The results are shown in figure 9 for the average PSF size. In this figure, the results are given in the curved detector's coordinate plane to facilitate comparison. The area of interest, determined by the dimensions of the optical chamber, is indicated by a cyan circle. Our target for a good cortical imaging instrument is to obtain a spot size no larger than approximately $50 \mu \mathrm{m}$, in order to resolve cortical columns. This criteria is met by the new curved detector instrument over most of the field view. 


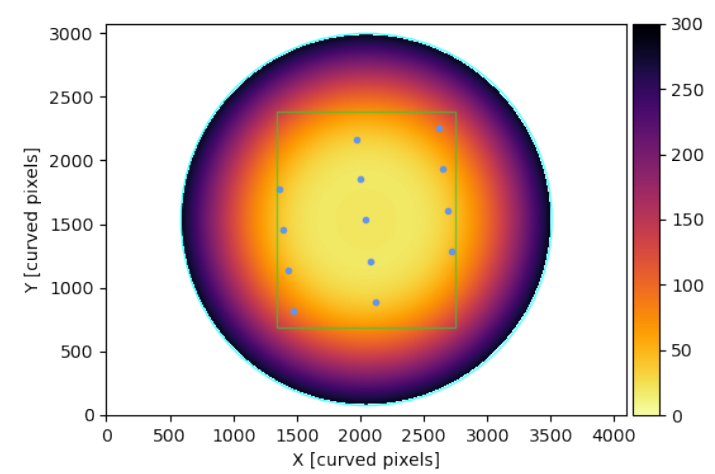

(a) Flat detector setup

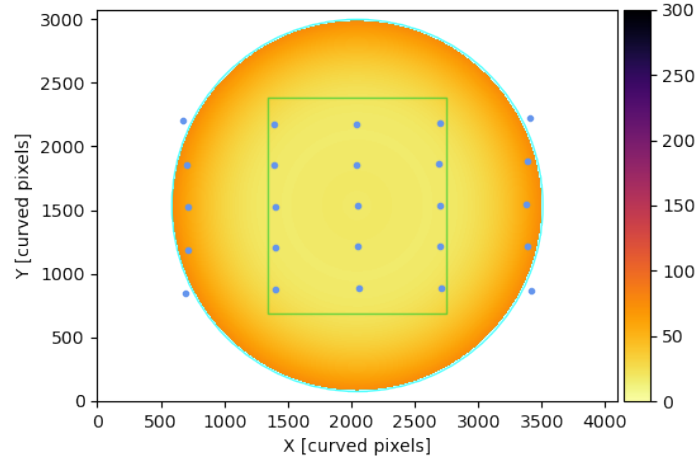

(b) Curved detector setup

Figure 9: Values of the average PSF's full width half $\max$ (FWHM) for both the curved and flat sensor setups. The PSFs have been measured at discrete points (blue dots) and from these images the FWHM was extracted. The average FWHM size between these points was estimated by fitting a polynomial function to the data, imposing radial symmetry. The coordinates and dimensions of the original flat imaging system's PSFs were converted to the curved imaging system coordinates such that both results are shown in the same reference frame to ease comparison. The dimensions of the optical chamber $(\mathrm{R}=9 \mathrm{~mm})$ is shown by a cyan circle. The flat sensor's area is indicated by a light green rectangle.

\subsection{Edge Sharpness Analysis}

The point spread function provides an unambiguous way of characterizing the optical setup. It remains however tedious to carry out, in part due to the requirement to move the pinhole along a spherical surface, and can not be performed outside of the optical test bench. In an attempt to obtain an easier to measure estimate of our optical instrument's performance, we developed an alternative method by calculating the edge sharpness in the acquired images. Although less precise than PSF measurements, this method has the advantages of being quick and easy, and allows us to compare images acquired on the optical test bench to experimental images acquired in vivo at INT, where the vascular pattern provides the sharp edges to be imaged.

The basic idea is simple: to measure how sharp the edges in the images are, where blurry images have softer edges than high quality images. Our algorithm is based on the sobel operator algorithm from the Python OpenCV library ${ }^{11}$ which computes a numerical derivative along a given axis. We obtain the gradient at each pixel in the image this way, knowing that the value of the gradient is proportional to the sharpness of the edge. This value in itself however does not have any physical meaning, as it depends on multiple factors, including the algorithm's kernel size. To confer significance to the sobel value, we introduce a small artificial feature in the image before computing the gradient. This artificial feature aims at simulating a perfect edge and consists of a white square in a larger black square. When the sobel algorithm is computed on this modified image, the highest value of sobel will be caused by the artificial perfect edge, and the sobel map of the whole image is then normalized by this value. As a result, a sobel of 1 always corresponds to a perfect edge (going from 0 to the full bit depth in one pixel), no matter the image considered or the kernel size. The image and the computed sobel map is then split into cells of fixed size: the cells must be large enough to include features of the image that can be detected by the algorithm but small enough that change in image quality within the cell is still negligible. The overall sharpness of a given cell is then determined by identifying the highest sobel value inside that cell.

Dust particles on the sensor create very sharp edges that distort results. Cleaning the detector is a very delicate task (because it is curved, there is no protective glass on the sensor) and requires pulling apart the setup. As a consequence, to avoid the dust issue we create a mask using a white image (obtained by imaging an illuminated piece of clean white paper for example), where the only discernible elements in the image are the dust particles. The resulting mask is thereafter applied to all images that are analysed in order to filter out the effect of pixels covered in dust.

The sobel value of an edge depends both on the blurring (by aberrations) and the contrast in the image 
(mostly caused by illumination variations). Since we are mostly concerned by the blurring of the image, we wish to decouple both effects in order to obtain a concrete estimate of the blurring, independently of the illumination. To that end, we have investigated the dependence of the sobel value for different combinations of greyscale contrast and blurring by manipulating a simulated image. The image was computer generated to represent an idealized version of the image used for testing (black dots on a white background). The contrast was tweaked by manipulating the greyscale levels of the image and the blurring was simulated by convoluting the simulated image with a Gaussian filter of width sigma. For each combination, the sobel value was computed and the overall results were tabulated. This is shown in figure 10 for various greyscale and blurring levels. A polynomial fit relating the blurring factor to the sobel value and the greyscale level can then be extracted. This provides a way to estimate the blurring in each image (or sub image) in physical units (pixels, $\mu \mathrm{m}$ ) by first computing the peak sobel value and the greyscale level of the image and then either looking up the combination of sobel and greyscale in the tabulated values or feeding them to the fit function, resulting in an estimate of the blurring.

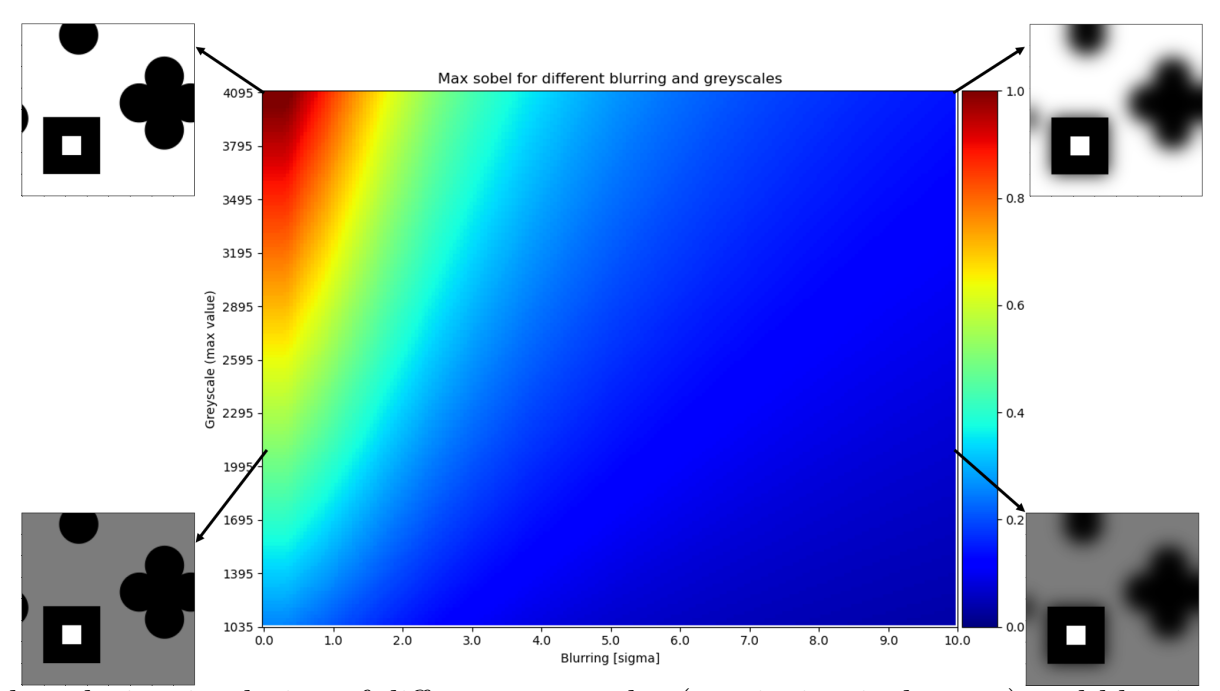

Figure 10: Sobel analysis: simulation of different greyscales (y-axis, in pixel count) and blurring widths (x-axis, in pixels) and the corresponding sobel value (colour map). The simulated image for four specific combination of greyscale and blurring are also shown in the figure, with black arrows pointing to the corresponding point in the plot for each image. The artificial perfect edge introduced to normalize the sobel values can be seen in these sample simulated images.

For consistency between the two setups (original flat and curved), all dimensions related to the sobel analysis (the dimensions of the artificial feature, the size of the cells and the size of the kernel used by the sobel algorithm) are defined in physical units (in this case, $160 \mu \mathrm{m}, 1.8 \mathrm{~mm}$ and $55 \mu \mathrm{m}$ respectively, although these can be adjusted). These values are then converted to pixels for each setup using the appropriate pixel size and magnification of the optics. This way, the results obtained on both the curved and flat detector setup can be compared to each other.

An example of a full sobel analysis is shown in figure 11. In this example, the test object is one of our 3D printed sphere, painted white and covered in black dots. The resulting sigma values in the region of interest, around $25 \mu \mathrm{m}$, are on the same order of magnitude as those extracted from the PSFs measured in the field of view of the curved detector setup.

The sobel algorithm has inherent flaws and is in no way intended to replace the more precise and accurate methods of quantifying an optical system. One such major flaw is that the blurring estimate assumes that the convoluting PSF is Gaussian, which it is not, and symmetric, which is also not the case in general. It does however offer an order of magnitude estimate that can be calculated quickly (in a few seconds) with any image that contains features with sharp edges. 


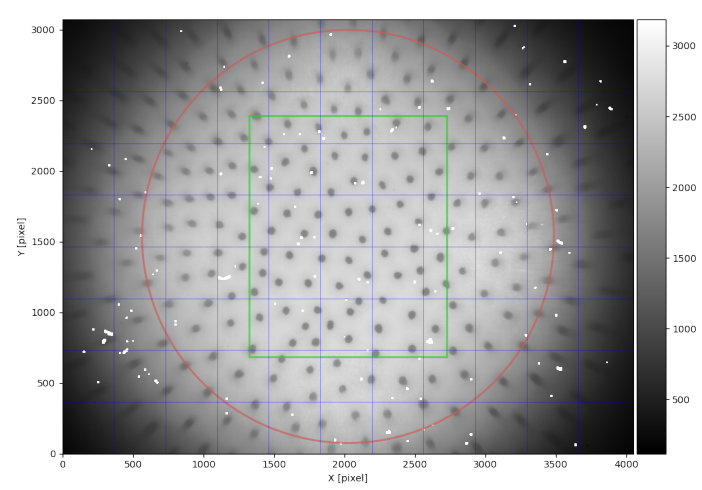

(a) Image

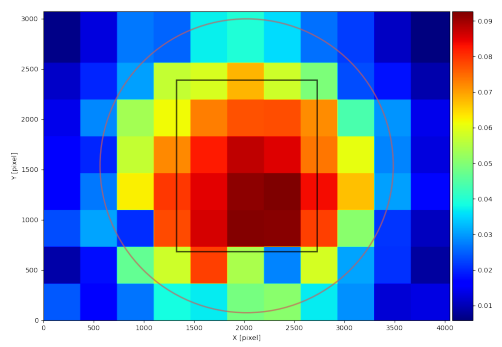

(c) Maximum Sobel per cell

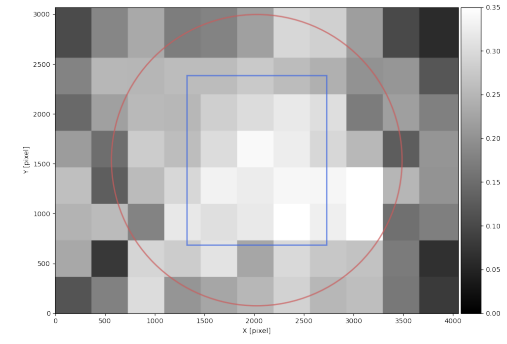

(d) Greyscale level per cell

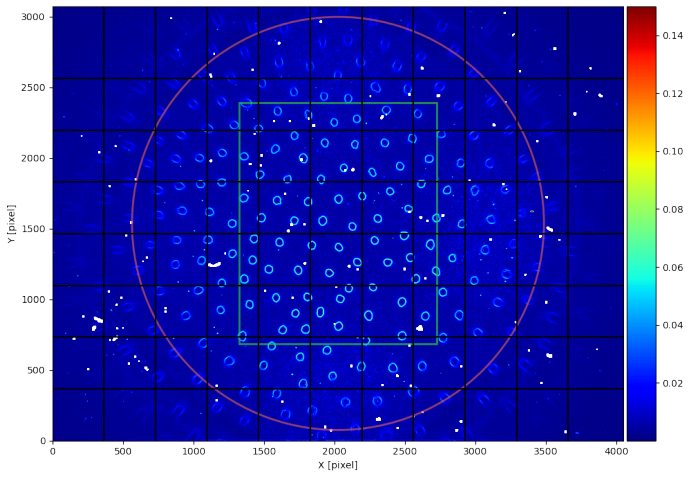

(b) Sobel

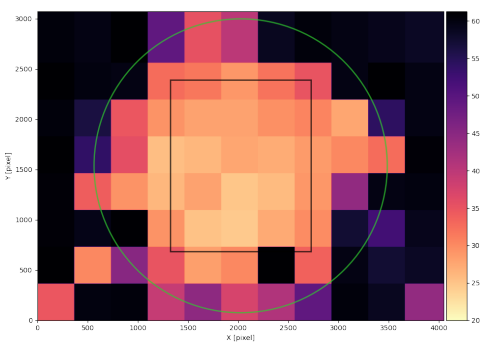

(e) Blurring per cell

Figure 11: Sobel analysis applied to an image of a white sphere covered with black dots obtained with the curved sensor instrument. In all figures the large circle indicates the size of the optical chamber and the smaller square indicates the area of the flat detector. The masked dust pieces appear white on the images. Figure a shows the original image, figure b shows the sobel map, obtained by applying the sobel algorithm to the image (kernel size $=11$ pixels), figure $\mathrm{c}$ shows the maximal sobel value for each cell (366x366 pixels per cell). Figure d shows the greyscale level for each cell (the maximum pixel value - the minimum pixel value) and figure e shows the blurring level computed from the greyscale and maximum sobel, for each cell. The blurring is expressed as sigma (in micrometers), where sigma here corresponds to the width of a Gaussian blurring matrix. The cells delimitation are drawn in black on top of the image and sobel maps.

\section{ILLUMINATION}

The illumination is a crucial part of the imaging system. An ideal illumination system would provide uniform illumination over the whole optical chamber at several different wavebands: red for VSD excitation, orange for intrinsic imaging, green to image the vascularization. The current illumination system in use at INT, based on a white halogen lamp used in combination with a shutter, optical fibres and various filters, is adequate. It however produces a lot of heat, and the uniformity and consistency of the illumination pattern for intrinsic imaging is limited by the use of manually placed optical fibres.

In an attempt to improve the illumination, we have designed and fabricated a new illumination system. This new system is made of four LED units (LZ4-00MA00 from LED Engine); the use of LEDs instead of a halogen lamp dramatically reduces the heat generated by the light sources. Each of these LED units is composed of four LEDs at 4 different wavelengths $(621 \mathrm{~nm}, 590 \mathrm{~nm}, 525 \mathrm{~nm}$ and $460 \mathrm{~nm})$. The blue light, although not a frequency that we normally use, can be useful for wide field calcium imaging with genetically encoded calcium indicators (GCaMP). The LEDs are uniformly disposed on a brass ring and fixed to brass wedges angled at $35^{\circ}$ towards the chamber. This ring is designed to be mounted on a $60 \mathrm{~mm}$ cage system from Thorlabs such that it fits well with the optical mount (as described in section 2.2). Figure 12 shows images of the resulting LED ring lit at different wavelengths. The LED ring has its own power supply and its electronic controls include a potentiometer for each colour that lets users set the illumination intensity independently. It also takes trigger inputs for each wavelength such that the LED ring can be smoothly integrated to the experimental procedure 
at INT.

In order to test the LED illumination ring, it was brought to INT and fixed to the existing imaging system. The resulting setup was used to gather images in vivo on the primary visual cortex of a macaque. Similar images were recorded a few days prior with the original halogen lamp for comparison. The recorded data includes multiple trials, and each trial consists of several images taken $10 \mathrm{~ms}$ apart, for about one second of recording for each trial. To investigate the change in illumination uniformity, the average intensity of these images was computed as a function of the distance away from the centre. This is shown in figure 13, where the average intensity produced by the LED ring is indeed more uniform than the one produced by the halogen lamp. The effect is predominantly visible on the edges of the image, as expected.

The use of LEDs not only decreases the generated heat and increases the illumination uniformity, but it also improves the stability of the produced light. This was confirmed by calculating the average pixel intensity in any given image for several trials and different time frames (using the same data that was used to investigate the illumination uniformity), as shown in figure 14. The measured intensity variation of the light emitted from the LED ring is found to be smaller than the variation observed for the halogen lamp illumination by a factor of 2.7. The illumination produced by the LED ring however exhibits a systematic spiking behaviour absent from the halogen illumination, which we suspect is caused by the LED current driver and might be reduced by using a different, more stable driver.

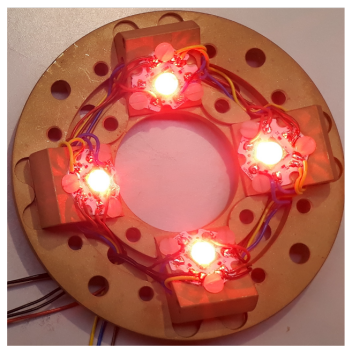

(a) Red $(621 \mathrm{~nm})$

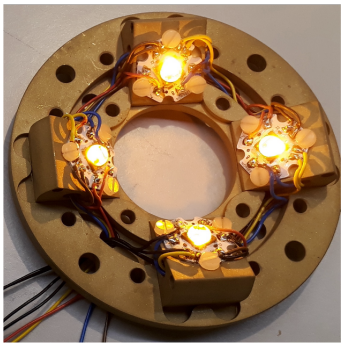

(b) Amber (590 nm)

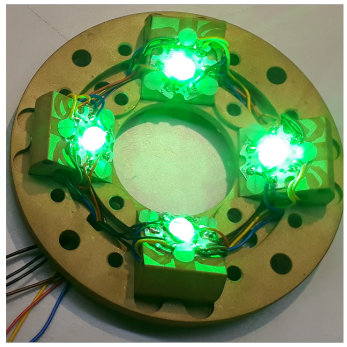

(c) Green $(525 \mathrm{~nm})$

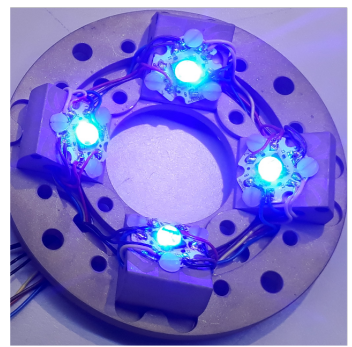

(d) Blue (460) nm

Figure 12: Illumination system: the four colours produced by the LED ring.

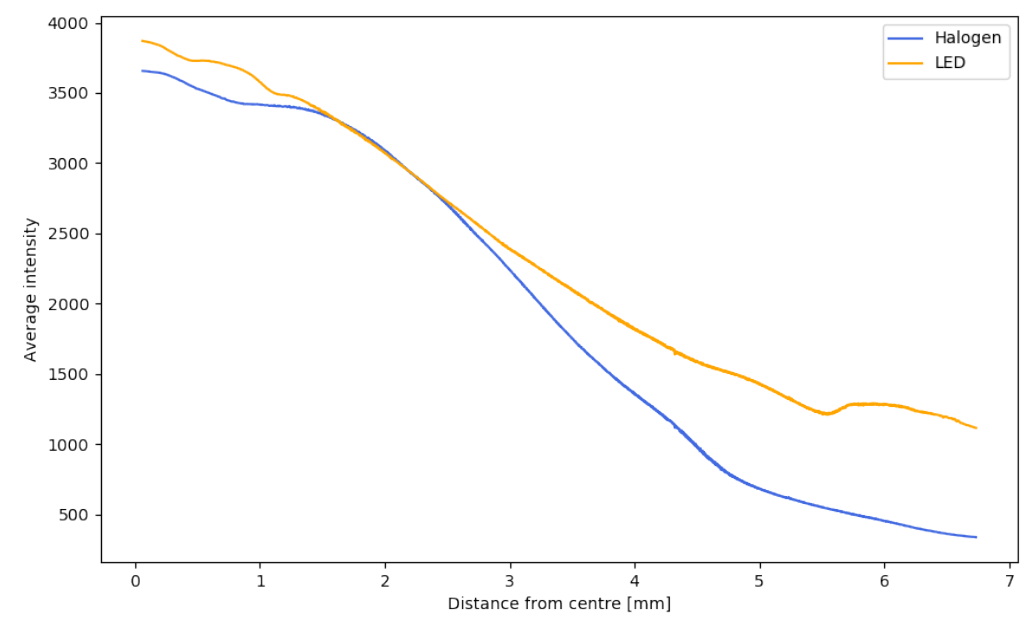

Figure 13: Light intensity (pixel value) as a function of the distance from the centre for halogen and LED illumination, averaged over all time frames for 10 trials of in vivo cortical imaging of macaque cortex. 


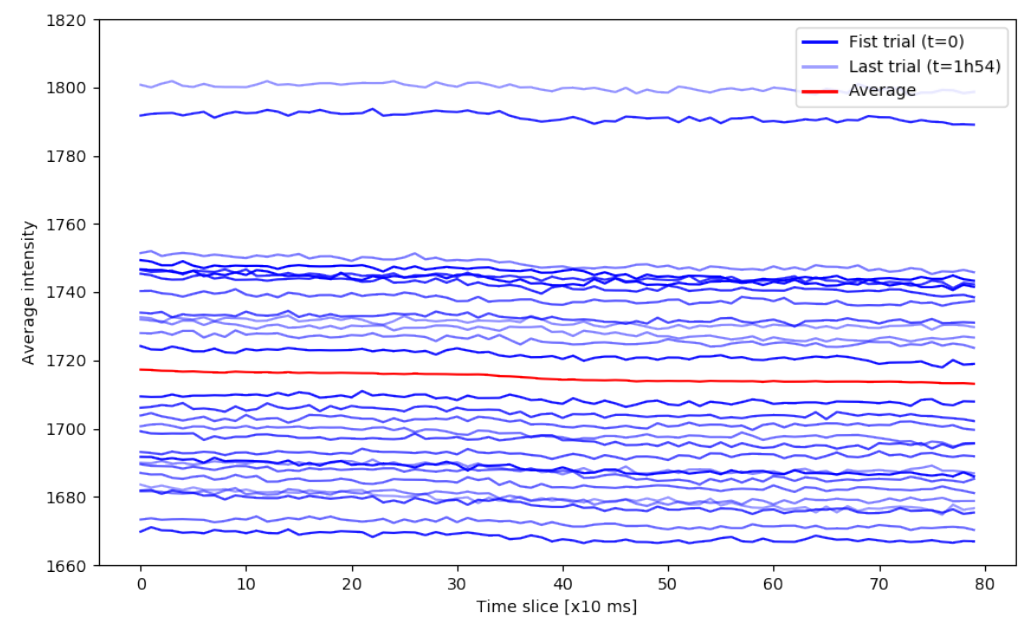

(a) Halogen lamp - 28 trials, std $=33.57$

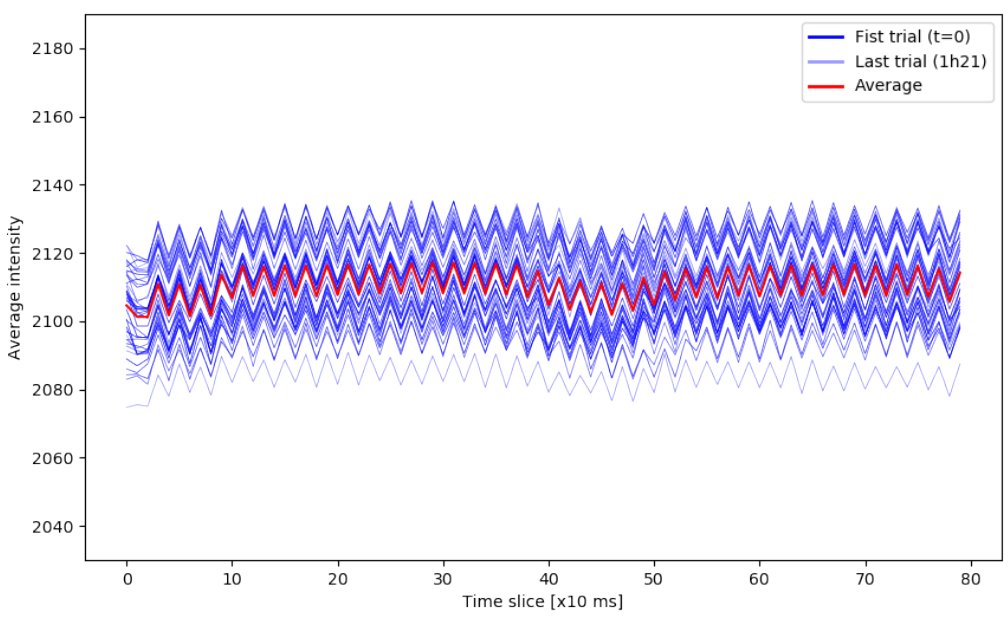

(b) LED ring - 40 trials, std $=12.23$

Figure 14: Average light intensity from cortical images obtained in vivo at each time frame for multiple trials, for the two illumination systems (Halogen lamp and LED ring). In both figures, each blue curve represents a single trial, with the darkness of the blue proportional to the time elapsed since the first trial (the first trial is the darkest and the last trial is the lightest). The average value for each time frame is plotted in red. The sample standard deviations are 33.57 and 12.23 for the halogen lamp and the LED ring, respectively. The data for both cases were taken a few days apart on the same setup and on the same monkey at INT.

\section{FUTURE WORK}

The optical setup is currently ready to be tried out at INT; however some technical issues concerning the data transmission speed achievable with the apertus ${ }^{\circ}$ AXIOM Beta camera for full size, full depth images has prevented us from doing so. A solution is being worked out in collaboration with apertus, and our short term goal is to attempt VSD imaging on a rhesus macaque's visual cortex.

Our long term goal is to adapt the optical system to the marmoset brain. The current imaging instrument is designed for the macaque brain for two main reasons: the larger brain makes the design easier, and macaques 
are by far the most accessible to us for in vivo testing. However marmosets have a lissencephalic (smooth) brain, which means that unlike macaques, none of the marmoset's cortical surface is hidden in sulci (brain folds). In theory we could therefore image all of the marmoset's cortical surface, given the proper optical tool. The difficulty in doing so lies in the fact that the marmoset is much smaller than the macaque (its brain is roughly 12 times smaller in volume than the macaque's ${ }^{12}$ ). To properly account for this new, smaller curvature will require new optics and possibly a new, more curved, detector.

In the meantime, we also aim to improve the imaging performance by developing a dynamic solution to account for the brain physiological pulsations created by breathing and heartbeat, either through post-processing methods or with active/adaptive optics, if necessary.

\section{ACKNOWLEDGMENTS}

We are grateful to Herbert Poetzl from apertus who has provided invaluable help in our dealings with the apertus $^{\circ}$ AXIOM Beta Developer Kit. We also thank Thibault Behaghel, who curved the detectors for this project, and Emmanuel Hugo and Kelly Joaquina who have contributed advice and help with the detector when it was damaged. Manon Bourbousson participated in the LED ring design and the early stages of the project. Finally, we thank Sandrine Chemla, who has provided help and many insights into the neuroscience experimental requirements and the INT setup.

The work presented in this paper was funded by CNRS 80 prime and is part of the ATTRACT programme that has received funding from the European Union's Horizon 2020 Research and Innovation programme under Grant Agreement N 777222 .

\section{REFERENCES}

[1] Grinvald, A. and Hildesheim, R., "Vsdi: a new era in functional imaging of cortical dynamics," Nature Reviews Neuroscience 5, 874-885 (2004).

[2] Chemla, S., Muller, L., Reynaud, A., Takerkart, S., Destexhe, A., and Chavane, F., "Improving voltagesensitive dye imaging: with a little help from computational approaches," Neurophotonics 4(3), 1 - 12 (2017).

[3] Chemla, S., Reynaud, A., di Volo, M., Zerlaut, Y., Perrinet, L., Destexhe, A., and Chavane, F., "Suppressive traveling waves shape representations of illusory motion in primary visual cortex of awake primate," The Journal of Neurosciences 39, 4298-4298 (2019).

[4] Muller, L., Chavane, F., Reynolds, J., and Sejnowski, T., "Cortical travelling waves: mechanisms and computational principles," The Journal of Neurosciences 19, 255-268 (2018).

[5] Zanos, T. P., Mineault, P. J., Nasiotis, K. T., Guitton, D., and 4, C. C. P., "A sensorimotor role for traveling waves in primate visual cortex," Neuron $\mathbf{8 5}, 615-627$ (2015).

[6] Rubino, D., Robbins, K. A., and Hatsopoulos, N. G., "Propagating waves mediate information transfer in the motor cortex," Nature Neuroscience 9(12), 1549-1557 (2006).

[7] Davis, Z. W., Muller, L., Martinez-Trujillo, J., Sejnowski, T., and Reynolds, J. H., "Spontaneous travelling cortical waves gate perception in behaving primates," Nature 587(7834), 432-436 (2020).

[8] Prechtl, J. C., Cohen, L. B., Pesaran, B., Mitra, P. P., and Kleinfeld, D., "Visual stimuli induce waves of electrical activity in turtle cortex," Proceedings of the National Academy of Sciences 94(14), 7621-7626 (1997).

[9] Muller, L., Reynaud, A., Chavane, F., and Destexhe, A., "The stimulus-evoked population response in visual cortex of awake monkey is a propagating wave," Nature Communications 5(1), 3675 (2014).

[10] Bourbousson, M., Racicot, I., Muslimov, E., Behaghel, T., Blaize, K., Bourdet, A., Chemla, S., Hugot, E., Jahn, W., Roux, S., Vanzetta, I., Weber, P., Sauvage, J.-F., Chavane, F., and Ferrari, M., "Imaging multiple cortical areas with high spatio-temporal resolution using innovative wide-field imaging system," in [Neurophotonics], Pavone, F. S., Cognet, L., and Kuner, T., eds., 11360, 1 - 11, International Society for Optics and Photonics, SPIE (2020).

[11] Bradski, G., "The OpenCV Library," Dr. Dobb's Journal of Software Tools (2000). 
[12] Van Essen, D. C., Donahue, C. J., Coalson, T. S., Kennedy, H., Hayashi, T., and Glasser, M. F., "Cerebral cortical folding, parcellation, and connectivity in humans, nonhuman primates, and mice," Proceedings of the National Academy of Sciences 116(52), 26173-26180 (2019). 